Chin. J. Astron. Astrophys. Vol. 0, No. 0, (200x) 000-000

Chinese Journal of

Astronomy and

Astrophysics

\title{
Strange stars: Can their crust reach the neutron drip density?
}

\author{
Hai Fu and Yong-Feng Huang \\ Department of Astronomy, Nanjing University, Nanjing 210093, China
}

ChJAA in press

\begin{abstract}
Electrostatic potential of electrons near the surface of static strange stars at zero temperature is studied within the frame of the MIT bag model. We find that for QCD parameters within rather wide ranges, and if the nuclear crust on the strange star is at a density leading to neutron drip, the electrostatic potential is insufficient to establish an outwardly directed electric field, which is crucial for the survival of such crusts. If a minimum gap width of $200 \mathrm{fm}$ is called in for a more stringent constraint, our calculations completely rule out the possibility of such crusts' presence on strange stars. Therefore, our results prefer against the existence of neutron-drip crust in nature.
\end{abstract}

Key words: dense matter - elementary particles - stars: neutron

\section{INTRODUCTION}

There may exist a more stable state of hadrons than ${ }^{56} \mathrm{Fe}$, called strange quark matter (SQM), which is a bulk quark phase consisting of roughly equal numbers of $u, d$, and $s$ quarks plus a smaller number of electrons to guarantee charge neutrality (Witten 1984). This theoretical result is unlikely to be proved or disproved through QCD calculations at least in the foreseeable future. Final adjudication must come from experiments conducted with accelerators or from astrophysical tests. One of the important consequences of Witten's hypothesis is the prediction of strange stars (Haensel, Zdunik, \& Schaeffer 1986; Alcock, Farhi, \& Olinto 1986, hereafter AFO), i.e., stars made of SQM. The presence of such stars should never be rare, in fact some authors even inferred that all neutron stars might have been converted to strange stars, since the whole Galaxy is likely to be contaminated by stranglets (Glendenning 1990; Madsen \& Olesen 1991; Caldwell \& Friedman 1991; Medina-Tanco \& Horvath 1996). For decades, unremitted efforts have been made to observationally discriminate strange stars from neutron stars, and some candidates for strange stars have been accumulating (Cheng et al. 1998; Li et al. 1999; $\mathrm{Xu}$ et al. 2001a, b). However, even to now it is still premature to reach any firm conclusions.

$\star$ E-mail: hyf@nju.edu.cn 
A strange star can have a normal matter crust. The density at the base of the crust is a crucial parameter, which has been discussed by many authors (AFO; Huang \& Lu 1997a, b; Xu \& Qiao 1999; Yuan \& Zhang 1999; Chen \& Zhang 2001; Ma et al. 2002). Recently, Ma et al. (2002) studied the influence of some parameters, such as the strange quark mass $\left(m_{s}\right)$, the bag constant $(B)$, and the strong coupling constant $\left(\alpha_{c}\right)$, on the crust density. However, their study does not give out clearly the ranges of parameters that enable a crust as dense as neutron drip density. Here we study the problem in more detail. The parameter ranges that support a neutron-drip-density crust will be calculated numerically. Our paper is arranged as follows. Section 2 describes the existence of strange star crust. Section 3 gives the detailed procedure of our calculations and the numerical results in the $\alpha_{c}-m_{s}-B$ space. Finally, we summarize the results in Section 4.

\section{THE EXISTENCE OF CRUST}

It was pointed out by AFO that a strange star could be covered by a normal material crust. The presence of electrons in SQM is vital to the existence of such crusts. Because $s$ quark's mass is larger than $u$ and $d$ quarks, it is slightly deficient in equilibrium SQM. Electrons are thus called in to make the system electrically charge neutral. As quarks are bounded through strong interaction, they should have a very sharp surface with thickness of the order of $1 \mathrm{fm}$. On the other hand, the electrons, bounded by the Coulomb force, can extend several hundred fermis beyond the quark surface. So, a strong electric field, $\sim 10^{17} \mathrm{~V} \mathrm{~cm}^{-1}$ and outwardly directed, will be established in a thin layer of several hundred fermis thick above the strange matter surface. This field can support a crust, composed of normal nuclear matter, suspended out of contact with the SQM core.

The so-called neutron drip density, $\rho_{\text {drip }} \sim 4.3 \times 10^{11} \mathrm{~g} \mathrm{~cm}^{-3}$ sets an absolute upper limit for the density of the crust, $\rho_{\text {crust }}$. The reason is that, if $\rho_{\text {crust }}$ reaches $\rho_{\text {drip }}$, neutrons will begin to drip out. Being electrically neutral, the neutrons will fall freely into the core, and, by hypothesis, be deconfined to be SQM. As a consequence, $\rho_{\text {crust }}$ will keep going down, until it's below $\rho_{\text {drip }}$. Conventionally, when the nuclear crust is taken into account, the bottom density was assumed to be $\rho_{\text {drip }}$. However, is neutron drip the only limit on the crust density? Could the crust density actually go so far? Huang \& Lu (1997a, b) said NO to both questions. By proposing that mechanical balance should be held between electric and gravitational forces on the whole crust, not only on a single nucleus as modelled by former authors (AFO), they claimed that at a density still far lower $\left(\sim \rho_{\text {drip }} / 5\right)$ than the neutron drip density, the crust would begin to break down.

AFO proposed a model to describe the gap between the SQM core and the nuclear crust within the framework of Thomas-Fermi model. The electric field should be described with the following Poisson's equation:

$$
\frac{d^{2} V}{d z^{2}}= \begin{cases}4 \alpha\left(V^{3}-V_{\mathrm{q}}^{3}\right) /\left[3 \pi(\hbar c)^{2}\right], & z \leq 0, \\ 4 \alpha V^{3} /\left[3 \pi(\hbar c)^{2}\right], & 0<z \leq z_{\mathrm{G}}, \\ 4 \alpha\left(V^{3}-V_{\mathrm{c}}^{3}\right) /\left[3 \pi(\hbar c)^{2}\right], & z_{\mathrm{G}}<z,\end{cases}
$$

where $z$ is a space coordinate measuring the height above the quark surface, $\alpha$ is the finestructure constant, $V_{\mathrm{q}}^{3} / 3 \pi^{2} \hbar^{3}$ is the quark charge density inside the quark matter, $V$ is the electrostatic potential of electrons, $V_{\mathrm{c}}$ is the electron Fermi momentum deep in the crust, which represents the positive charge density of the ions within the crust, and $z_{\mathrm{G}}$ is the gap width between the SQM surface and the base of the crust. In fact, $V_{\mathrm{q}}$ and $V_{\mathrm{c}}$ are the boundary values of the above equation: $V \rightarrow V_{\mathrm{q}}$ as $z \rightarrow-\infty, V \rightarrow V_{\mathrm{c}}$ as $z \rightarrow+\infty$. Meaningful solutions to the 
above equations occur only if $V_{\mathrm{c}}<V_{\mathrm{q}}$. Actually in a core-crust system, $V_{q}$ turns out to be the electron electrostatic potential at $R_{m}$. Here $R_{m}$ represents the maximum radius below which electrical charge neutrality is locally satisfied in the SQM core. Certainly $R_{m}$ is smaller than $R$, the radius of the core.

Since the electron chemical potential (we will prove that it equals to $V$ later) at which neutron drip occurs is $\sim 26 \mathrm{MeV}$ (Baym, Pethick, \& Sutherland 1971), to keep a crust at a density of $\rho_{\text {drip }}$ suspended, the electrostatic potential of electrons near the edge of the SQM core, $V_{q}$, must at least be larger than $26 \mathrm{MeV}$. In this work, we calculated $V_{q}$ for static SQM cores at zero temperature. Because of the uncertainties inherent in the critical QCD-related parameters, the $s$ quark mass, $m_{s}$, the strong interaction coupling constant, $\alpha_{c}$, and the bag constant, $B$, our calculations were actually carried out in a space expanded by those three parameters. We found that for parameters within rather wide ranges, to support a crust at a density leading to neutron drip is not possible. Especially, for the conventional choice of the parameters, i.e., $m_{s}=200 \mathrm{MeV}, \alpha_{c}=0.3$, and $B^{1 / 4}=145 \mathrm{MeV}$, our calculations indicate $V_{q} \simeq 20$ $\mathrm{MeV}$, well below $26 \mathrm{MeV}$.

It is interesting to mention that the properties of charm-quark stars (viz. strange-quark stars with an additional charm-quark population) have been studied by Kettner et al. (1995). But unfortunately, they found charm-quark stars are unstable against radial oscillations, i.e., no such stars can exist in nature. So our work will only pivot around strange-quark stars.

\section{ELECTROSTATIC POTENTIAL OF ELECTRONS IN SQM CORES}

\subsection{Governing Equations}

The property of SQM is generally described using the phenomenological MIT bag model (Chodos et al. 1974), which simplifies the dynamics of confinement by introducing an approximation that the quarks are separated from the vacuum by a phase boundary and the region in which quarks live is endowed with a constant universal energy density $B$. Since the description of SQM has been introduced elsewhere (Farhi \& Jaffe 1984; Kettner et al 1995), we will go straightforward to the governing equations and describe the procedure of our calculations. Our goal is to determine the electrostatic potential of electrons at $R_{m}$, i.e., $V_{q}$.

We assume that charge neutrality is locally satisfied. This is true popularly: Due to the rearrangement of electron charge inside and outside of the surface of an SQM core, the redundant positive charge of the quarks will be balanced locally by $e^{-}$only up to radial distances $r \leq R_{m}$. However, $R_{m}$ in fact is only minutely smaller (several hundred fermis, see AFO; Kettner et al $1995)$ than $R$, the core's radius.

Basing upon the realization that a core-crust system's temperature is generally much smaller than the typical chemical potentials of the constituents $\left(u, d, s, e^{-}\right)$, we further assume the core is at zero temperature. And we include first-order $\alpha_{c}$ effects in our calculations.

The thermodynamic potentials (per unit volume) as functions of the chemical potentials of the constituents read (Farhi \& Jaffe 1984;AFO):

$$
\begin{aligned}
\Omega_{f}\left(\mu_{f}\right)= & -\frac{\mu_{f}{ }^{4}}{4 \pi^{2}(\hbar c)^{3}}\left(1-\frac{2 \alpha_{c}}{\pi}\right), \quad f=u, d, \\
\Omega_{s}\left(\mu_{s}\right)= & -\frac{1}{4 \pi^{2}(\hbar c)^{3}}\left\{\mu_{s}\left(\mu_{s}{ }^{2}-m_{s}{ }^{2} c^{4}\right)^{1 / 2}\left(\mu_{s}{ }^{2}-\frac{5}{2} m_{s}{ }^{2} c^{4}\right)+\frac{3}{2} m_{s}{ }^{4} c^{8} \ln \left(\frac{\mu_{s}+\left(\mu_{s}{ }^{2}-m_{s}{ }^{2} c^{4}\right)^{1 / 2}}{m_{s} c^{2}}\right)\right. \\
& -\frac{2 \alpha_{c}}{\pi}\left[3\left(\mu_{s}\left(\mu_{s}{ }^{2}-m_{s}{ }^{2} c^{4}\right)^{1 / 2}-m_{s}{ }^{2} c^{4} \ln \left(\frac{\mu_{s}+\left(\mu_{s}{ }^{2}-m_{s}{ }^{2} c^{4}\right)^{1 / 2}}{m_{s} c^{2}}\right)\right)^{2}-2\left(\mu_{s}{ }^{2}-m_{s}{ }^{2} c^{4}\right)^{2}\right. \\
& -3 m_{s}{ }^{4} c^{8} \ln ^{2}\left(\frac{m_{s} c^{2}}{\mu_{s}}\right)+6 \ln \left(\frac{\rho_{R}}{\mu_{s}}\right)\left(\mu_{s} m_{s}{ }^{2} c^{4}\left(\mu_{s}{ }^{2}-m_{s}{ }^{2} c^{4}\right)^{1 / 2}\right.
\end{aligned}
$$




$$
\begin{gathered}
\left.\left.\left.-m_{s}{ }^{4} c^{8} \ln \left(\frac{\mu_{s}+\left(\mu_{s}{ }^{2}-m_{s}{ }^{2} c^{4}\right)^{1 / 2}}{m_{s} c^{2}}\right)\right)\right]\right\}, \\
\Omega_{e}\left(\mu_{e}\right)=-\frac{\mu_{e}{ }^{4}}{12 \pi^{2}(\hbar c)^{3}} .
\end{gathered}
$$

The renormalization point for the SQM, $\rho_{R}$, which appears in Eq. (3), is chosen to be 313 $\mathrm{MeV}$ in this work, because of the reasons pointed out by AFO. The number densities for every constituent can be expressed in terms of $\mu_{i}(i=u, d, s, e)$ from (Farhi \& Jaffe 1984;AFO)

$$
n_{i}\left(\mu_{i}\right)=-\frac{\partial \Omega_{i}}{\partial \mu_{i}}
$$

and charge neutrality requires

$$
\frac{2}{3} n_{u}-\frac{1}{3} n_{d}-\frac{1}{3} n_{s}-n_{e}=0 .
$$

In fact, Eq. (6) could be rendered to an equation of $\mu_{i}\left(i=u, d, s, e^{-}\right)$if we substitute Eqs. (2) -(5) into it. Later in this section, we will demonstrate that all $\mu_{i}$ at a given energy density can be determined if combined with the chemical equilibrium conditions and the energy density equation, and we will point out that $V_{q}$ is equal to $\mu_{e}$.

Chemical equilibrium between the three quark flavors and the electrons is maintained by weak interactions (i.e., $\beta$-stable SQM, Farhi \& Jaffe 1984; AFO)

$$
\begin{aligned}
d & \rightarrow u+e+\bar{\nu}_{e}, \\
u+e & \rightarrow d+\nu_{e}, \\
s & \rightarrow u+e+\bar{\nu}_{e}, \\
u+e & \rightarrow s+\nu_{e},
\end{aligned}
$$

and

$$
s+u \leftrightarrow d+u .
$$

The loss of neutrinos by the star implies that their chemical potential is equal to zero (we ignore the effect brought by the finite mass of neutrinos). Hence, at equilibrium the chemical potentials should obey:

$$
\begin{aligned}
& \mu_{d}=\mu_{s} \equiv \mu, \\
& \mu_{u}+\mu_{e}=\mu .
\end{aligned}
$$

Combined with the condition of charge neutrality (Eq. (6)), these equations leave us with only one independent chemical potential, which we have denoted by $\mu$ (see Eq. (12)). Therefore, we need still one equation to make the system close.

The total energy density $\rho$ is given by

$$
\rho=\sum\left(\Omega_{i}\left(\mu_{i}\right)+\mu_{i} n_{i}\left(\mu_{i}\right)\right)+B, \quad i=u, d, s, e^{-} .
$$

Since all $\mu_{i}$ could be expressed in terms of $\mu$, solving Eq. (14) can determine $\mu$ if given the value of $\rho$, and so forth, $\mu_{i}$.

If the Thomas-Fermi model is invoked to describe the electrons associated with the quarks, we could easily arrive at the conclusion that $V=\mu_{e}$, i.e., the electrostatic potential is equal to the chemical potential for the electrons (see, e.g., AFO; Kettner et al 1995). The derivation is as follows. The number density of electrons is given by the local Fermi momentum $P_{e}$,

$$
n_{e}=P_{e}^{3} /\left(3 \pi^{2} \hbar^{3}\right) .
$$


On the other hand,

$$
n_{e}=-\partial \Omega_{e} / \partial \mu_{e}=\mu_{e}^{3} /\left(3 \pi^{2} \hbar^{3} c^{3}\right) .
$$

From the above two equations, we get $\mu_{e}=P_{e} c$. Since the electrons are confined within a sphere of infinite radius, their total energy $-V+P c$ should obey, $-V+P c \leq-V(\infty)=0$, i.e., $-V+P_{e} c=0$, and so forth,

$$
V=P_{e} c=\mu_{e}
$$

As $\mu_{e}$ at $R_{m}$ could be calculated following the procedure stated above, $V_{q}$ is readily at hand.

\subsection{Numerical Results}

Since $\mu_{e}$ decreases with density (Kettner et al 1995), which reflects the fact that less electrons are needed in denser SQM, the electrostatic potential of electrons increases monotonically from the center toward the surface of strange stars. Because when $r<R_{m}$ the electrical charge neutrality is locally satisfied, only the electron electrostatic potential at $R_{m}$, corresponding to $V_{q}$ in Eq. (1) is responsible for supporting the nuclear crust. Owing to the equation of state $P=(\rho-4 B) / 3$ (Witten 1984) ${ }^{1}$, the energy density at the surface of SQM cores is universally equal to $4 B$, independent of the star's mass, or in other words, of the central density. Hence, although in our calculations we fixed the energy density at $4 B$, our results will stand for the complete equilibrium sequences of compact SQM core-crust systems as determined by Glendenning, Kettner, \& Weber (1995).

There exist large uncertainties in the three QCD-related parameters: the $s$ quark mass, $m_{s}$, the strong interaction coupling constant, $\alpha_{c}$, and the bag constant, $B$. Our calculations are actually carried out in a space expanded by those parameters. The exact values of them are unknown but are probably constrained within: $50 \mathrm{MeV} \leq m_{s} \leq 340 \mathrm{MeV}, 0 \leq \alpha_{c} \leq 0.6$, and $135 \mathrm{MeV} \leq B^{1 / 4} \leq 165 \mathrm{MeV}$.

The contours at fixed $V_{q}$ in the $\alpha_{c}-m_{s}$ plane for $B^{1 / 4}=135,145,155,165 \mathrm{MeV}$ are respectively presented in Fig. 1(a)-(d). The solid and dashed curves refer to $V_{q}=26 \mathrm{MeV}$ (the electron chemical potential at which neutron drip occurs) and $V_{q}=0 \mathrm{MeV}$, respectively. The gray regions show where the energy per baryon of the SQM, $\mu_{n}\left(\mu_{n} \equiv \mu_{u}+\mu_{d}+\mu_{s}\right)$, exceeds the lowest energy per baryon found in nuclei, which is $930 \mathrm{MeV}$ for ${ }^{56} \mathrm{Fe}$. The black regions are regions of no physical solutions.

To support a crust at a density leading to neutron drip, $V_{q}$ must, at least, be larger than $26 \mathrm{MeV}$ (in order to establish an outwardly directed field, or in other words, to get meaningful solutions to Eq. (10). So, only parameters in regions above the solid curves can make such crusts possible, and, of course, they should avoid the gray and black regions. Conventionally, the three parameters are chosen to be $m_{s}=200 \mathrm{MeV}, \alpha_{c}=0.3$, and $B^{1 / 4}=145 \mathrm{MeV}$. The cross in Fig. 1(b) represents this set. Obviously, it lives outside the permitted region, and actually $V_{q}$ equals roughly $20 \mathrm{MeV}$. We point out emphatically that $V_{q}<26 \mathrm{MeV}$ here.

Fig. 1 clearly shows that for QCD parameters within rather wide ranges, owing to the insufficient electrostatic potential of electrons, an SQM core is not capable of carrying a crust at $\rho_{\text {drip }}$ suspended.

Another factor we should take into account is the gap width, $z_{G}$. Note that the lattice spacing in the crust is $\sim 200 \mathrm{fm}$, in the same order of $z_{G}$, while the model (i.e., Eq. (11) assumed a smooth distribution of the ionic charges in the crust. In order to make their analysis selfconsistent, AFO assumed that the width of the gap should be larger than $200 \mathrm{fm}$. $z_{G}$ is entirely determined by $V_{c}$ and $V_{q}$ in AFO's model, we thus can also determine $V_{q}$ if given the values

\footnotetext{
1 Although this EOS is derived in the limit $m_{s} \rightarrow 0, \alpha_{c} \rightarrow 0$, for intermediate values of $m_{s}$ this equation is less than $4 \%$ different from the full expression (AFO).
} 
of $z_{G}$ and $V_{c}$. If $z_{G}=200 \mathrm{fm}$ and $V_{c}=26 \mathrm{MeV}$ (viz. a neutron-drip crust), then $V_{q}$ was found to be $\sim 125 \mathrm{MeV}$ after solving Eq. (1) with the associated boundary conditions (i.e., $V \rightarrow V_{\mathrm{q}}$ as $z \rightarrow-\infty, V \rightarrow V_{\mathrm{c}}$ as $z \rightarrow+\infty$, and $V, d V / d z$ both continuous at $\mathrm{z}=0$ and at $\mathrm{z}=z_{G}$ ) as well as the approximation $d V / d z=0$ for $z \geq z_{G}$ (viz. the gravitation forces are neglected ${ }^{2}$ ). Therefore, if a gap width larger than $200 \mathrm{fm}$ is really a necessary qualification for the stability of a core-crust system, the assumed presence of crusts at neutron drip density would have been completely ruled out, since no QCD parameters can make $V_{q}$ so high, at least within the ranges in which our calculations were done.

Although our calculations were limited to SQM at zero temperature, we can state unambiguously that a finite temperature can only make such dense crusts' situation even worse. According to Kettner et al. (1995)'s calculations (see Fig. 12 in their paper), $V_{q}$ decreases with temperature of the SQM. It thus becomes even more unlikely that a neutron-drip crust can exist above strange stars at higher temperatures.

\section{CONCLUSIONS}

We have studied the electrostatic potential of electrons near the surface of SQM cores in a parameter space expanded by $m_{s}, \alpha_{c}$ and $B$, in order to evaluate the feasibility of the existence of crusts at neutron drip density on strange stars. Our numerical results indicate:

1. For QCD parameters within rather large ranges (Fig. 1), the electrons in an SQM core are incapable of establishing an outwardly directed electric field to carry a neutron-drip crust suspended.

2. If it is a sound criteria that the gap width $z_{G}$ should be larger than $200 \mathrm{fm}$, then the possibility of neutron-drip crust's presence on strange stars is completed ruled out by our calculations.

Therefore, our results prefer against the presence of such crusts in nature.

Acknnowledgements This work was supported by The Foundation for the Author of National Excellent Doctoral Dissertation of P. R. China (Project No: 200125), the Special Funds for Major State Basic Research Projects, and the National Natural Science Foundation of China.

\section{References}

Alcock C., Farhi E., Olinto A., 1986, ApJ, 310, 261 (AFO)

Baym G., Pethick C. J., Sutherland P. G., 1971, ApJ, 170, 299

Caldwell R. R., Friedman J. C., 1991, Phys. Lett. B, 264, 143

Chen C. X., Zhang J. L., 2001, Chin. Phys. Lett., 18, 145

Cheng K. S., Dai Z. G., Wei D. M., Lu T., 1998, Science, 280, 407

Chodos A., Jaffe R. L., Johnson K., Thorn C. B., Weisskopf V. F., 1974, Phys. Rev. D, 9, 3471

Farhi E., Jaffe R. L., 1984, Phys. Rev. D, 30, 2379

Glendenning N. K., 1990, Mod. Phys. Lett. A, 5, 2197

Glendenning N. K., Kettner Ch., Weber F., 1995, ApJ, 450, 253

Haensel P., Zdunik J. L., Schaeffer R., 1986, A\&A, 160, 121

Huang Y. F., Lu T., 1997a, Chin. Phys. Lett., 14, 314

Huang Y. F., Lu T., 1997b, A\&A, 325, 189

Kettner Ch., Weber F., Weigel M.K., Glendenning N.K. 1995, Phys. Rev. D, 51, 1440

Li X.-D., Bombaci I., Dey M., Dey J., vandenHeuvel E. P. J., 1999, Phys. Rev. Lett., 83, 3776

\footnotetext{
${ }^{2}$ Though this approximation has been criticized by Huang \& Lu (1997), it is accurate enough for our purpose here.
} 
Ma Z. X., Dai Z. G., Huang Y. F., Lu T., 2002, ApSS, 282, 537

Madsen J., Olesen M. L., 1991, Phys. Rev. D, 43, 1069; erratum: 44, 4150

Medina-Tanco G. A., Horvath J. E., 1996, ApJ, 464, 354

Witten E., 1984, Phys. Rev. D, 30, 272

Xu R. X., Qiao G. J., 1999, Chin. Phys. Lett., 16, 778

Xu R. X., Xu X. B., Wu X. J., 2001a, Chin. Phys. Lett., 18, 837

Xu R. X., Zhang B., Qiao G. J., 2001b, Astropart. Phys., 15, 101

Yuan Y. F., Zhang J. L., 1999, A\&A, 344, 371 

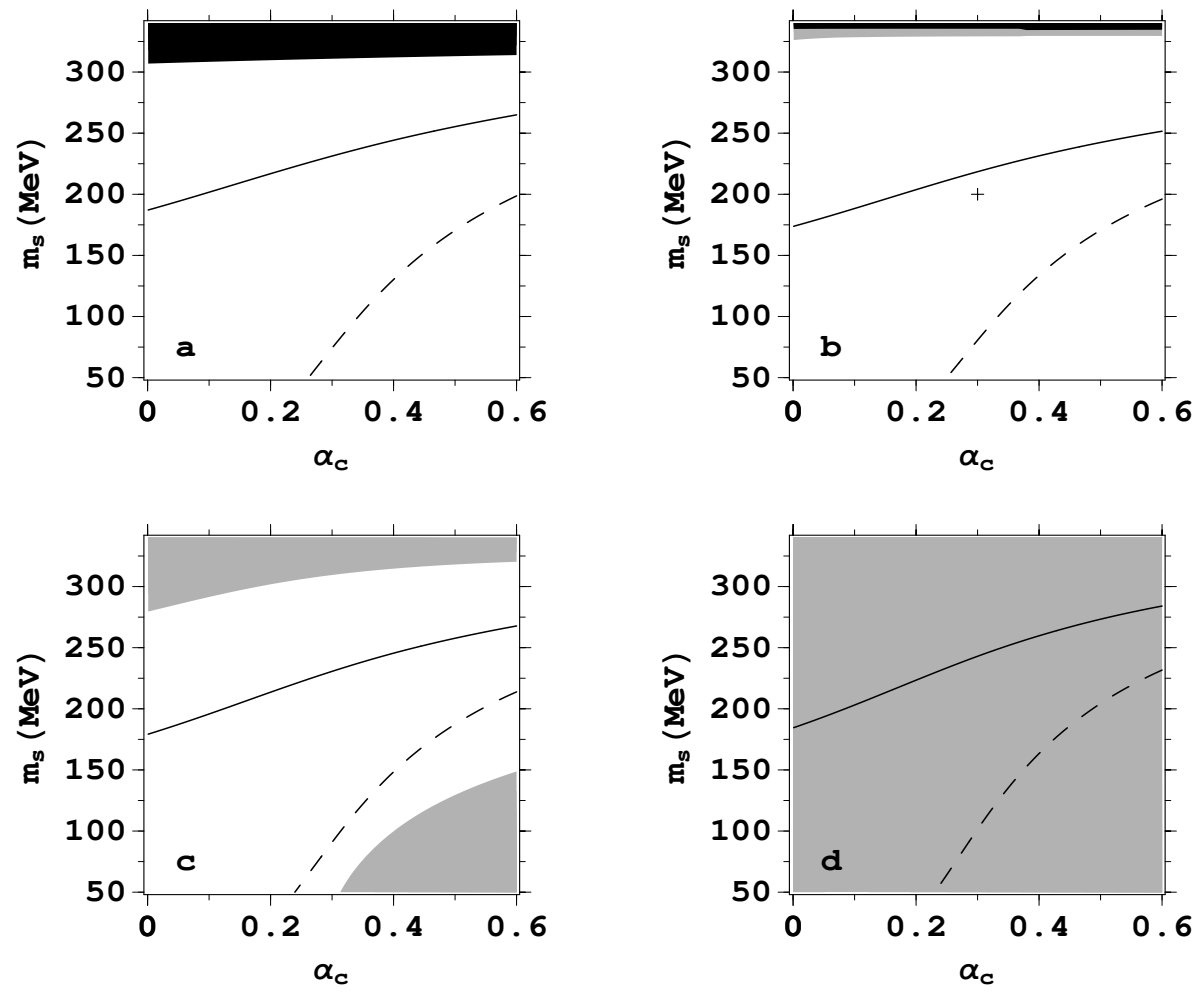

Fig. 1 Contours of fixed $V_{q}$ in $\alpha_{c}-m_{s}$ plane for $B^{1 / 4}=135,145,155,165 \mathrm{MeV}$. The cross near the center of (b) marks the position of conventional choice of QCD parameters, i.e., $m_{s}=200 \mathrm{MeV}, \alpha_{c}=0.3$, and $B^{1 / 4}=145 \mathrm{MeV}$. The solid and dashed curves refer to $V_{q}=26 \mathrm{MeV}$ (the electron chemical potential at which neutron drip occurs) and $V_{q}=0 \mathrm{MeV}$, respectively. The gray regions show where the energy per baryon of the SQM exceeds the lowest energy per baryon found in nuclei, which is 930 $\mathrm{MeV}$ for iron. The black regions are regions of no physical solutions. Obviously, for QCD parameters within rather wide ranges (including the conventional choice), SQM cores are incapable of supporting crusts at neutron drip density (see text for details). These results are independent of stellar mass, or in other words, of central density, because in the MIT bag model the energy density at zero pressure (corresponding to the surface of SQM cores) is universally equal to $4 B$. 